\title{
Surface localisation of master knot of Henry, in situ and ex vivo length of flexor hallucis longus tendon: pertinent data for tendon harvesting and transfer
}

\author{
P. Wan-ae-loh', P. Danginthawat ${ }^{2}$, T. Huanmanop ${ }^{3}$, S. Agthong ${ }^{3}$, V. Chentanez ${ }^{3}$ \\ 'PhD Candidate, Medical Science Programme, Faculty of Medicine, King Chulalongkorn Memorial Hospital, \\ Chulalongkorn University, Bangkok, Thailand \\ 2Faculty of Physical Therapy, Huachiew Chalermprakiet University, Samutprakan, Thailand \\ ${ }^{3}$ Department of Anatomy, Faculty of Medicine, King Chulalongkorn Memorial Hospital, Chulalongkorn University, \\ Bangkok, Thailand
}

[Received: 19 February 2020; Accepted: 30 March 2020]

Background: Length of flexor hallucis longus (FHL), localisation of master knot of Henry (MKH) and relationship between MKH and neurovascular bundle are essential for the achievement of FHL tendon transfer. The purpose of this study is to define the localisation of MKH in reference to bony landmarks of the foot, its relationship to plantar neurovascular bundle and to investigate in situ and ex vivo length of FHL tendon in single incision, double incision and minimally invasive techniques.

Materials and methods: Foot length was examined in 62 feet of 31 soft cadavers (9 males, 22 females). Various parameters including the relationship between MKH and neurovascular bundle, the distances from MKH to medial malleolus (MM), navicular tuberosity (NT) and the first interphalangeal joint of great toe (IP) were measured. Surface localisation of MKH in relation to a line joining the medial end of plantar flexion crease at the base of great toes (MC) to NT (MC-NT line) was determined. Lengths of FHL tendon graft from three surgical techniques were examined. In situ length was measured in the plantar surface of foot and ex vivo length was measured after tendon was cut from its insertion.

Results: The mean length of foot was $230.98 \pm 15.35 \mathrm{~mm}$ with a statistically significant difference between genders in both sides $(p<0.05)$. No distance was found between medial plantar neurovascular bundle (MPNVB) and MKH. Mean distance of $17.13 \pm 3.55 \mathrm{~mm}$ was found between lateral plantar neurovascular bundle (LPNVB) and MKH. MKH was located at a mean distance of $117.11 \pm 1.00 \mathrm{~mm}$ proximal to IP, $26.28 \pm 4.75 \mathrm{~mm}$ under NT and $59.58 \pm 7.51 \mathrm{~mm}$ distal to MM with a statistically significant difference of MKH-IP distance between genders in both sides and MKH-NT in right side. MKH was located anterior to NT (66.1\%), at NT (27.4\%) and posterior to NT (6.5\%) on the MC-NT line. Surface localisation of MKH was $94.75 \pm 8.43 \%$ of MC-NT line from MC with a perpendicular distance of $25.11 \pm 5.37 \mathrm{~mm}$ below MC-NT line. The in situ and ex vivo tendon lengths from MTJ to ST, to MKH and to IP were $39.05 \pm 10.88 \mathrm{~mm}$ and $34.43 \pm 10.23 \mathrm{~mm}$,

Address for correspondence: V. Chentanez, MD, PhD, Department of Anatomy, Faculty of Medicine, King Chulalongkorn Memorial Hospital, Chulalongkorn University, Bangkok 10330, Thailand, tel: 66-860701084, e-mail: fmedvct@gmail.com

This article is available in open access under Creative Common Attribution-Non-Commercial-No Derivatives 4.0 International (CC BY-NC-ND 4.0) license, allowing to download articles and share them with others as long as they credit the authors and the publisher, but without permission to change them in any way or use them commercially. 
$73.45 \pm 9.91 \mathrm{~mm}$ and $68.63 \pm 9.43 \mathrm{~mm}, 197.98 \pm 13.89$ and $191.79 \pm 14.00 \mathrm{~mm}$, respectively. A statistically significant difference between genders was found in MTJ-IP of in situ and ex vivo length of both sides $(p<0.05)$. The mean length of tendon between in situ and ex vivo was significantly different in all techniques $(p<0.05)$. A moderate positive correlation between foot length and tendon length was found in MTJ-IP of both in situ and ex vivo tendon length.

Conclusions: A statistically significant difference between in situ and ex vivo tendon length was shown in all harvesting techniques. Surface location of MKH was approximately at 95\% of MC-NT line from MC with a perpendicular distance of $25 \mathrm{~mm}$ from MC-NT line. (Folia Morphol 2021; 80, 2: 415-424)

Key words: flexor hallucis longus, master knot of Henry, tendon transfer

\section{INTRODUCTION}

Flexor hallucis longus (FHL) tendon transfer is a widely used technique for reconstruction of the Achilles tendinopathies $[17,20]$. This technique proposes to repair the length, to strengthen the injured tendon with additional tendon, and to corporate more muscle force to the plantar flexor [6]. FHL is appropriate for transfer because of its strength, axis and amplitude of contraction, and its concomitant action with triceps surae muscles [32]. Moreover, FHL transfer can also reduce the pain by normalising vascularity $[1,6]$. FHL transfer is also used for the treatment of posterior tibial insufficiency with a good to excellent clinical outcome $[8,19]$.

There are many techniques for harvesting $\mathrm{FHL}$ tendon grafts including single incision, double incision, and minimally invasive techniques [13]. The differences among these techniques are the indication and sites of incision. Importantly, the length of harvested tendon from each technique is vastly different [13]. The single incision approach is used to harvest the FHL within the tarsal tunnel. Although, this technique yields a shorter graft but it is long enough to be inserted on the calcaneus. When the additional length is required, the double incision technique at the medial aspect of foot near master knot of Henry (MKH) is considered. The added $3 \mathrm{~cm}$ of tendon length is obtained from this technique if the FHL is cut at MKH $[4,27,29]$. The minimally invasive technique provides the longest length [17]. Although, previous reports revealed good results following FHL transfer, complications such as serious injury of the distal branches of the posterior tibial artery and nerve, cock-up deformity, and functional loss of toe have been reported $[1,7,13,26]$.

Anatomically, medial and lateral plantar neurovascular bundles (MPNVB, LPNVB) reside near the incision line. In consequence, they might be at risk during harvesting. The other structure which affects tendon harvesting is MKH. MKH is where the tendon of flexor digitorum longus (FDL) crosses over the tendon of FHL. It has been used as a surgical landmark for the tendon graft harvesting $[2,17]$. Therefore, the precise location of the MKH is crucial for better results [2].

Knowledge of the length of $\mathrm{FHL}$ tendon available for harvesting, the relationship between tendon and neurovascular bundle, and the anatomical locations of MKH are essential for guiding the surgeon during operation and decreasing potential morbidity [13]. The length of FHL tendon after cutting for harvesting, which might be different from the length of attached tendon, should be elucidated. This anatomical study aims to clarify these issues in soft cadavers.

\section{MATERIALS AND METHODS}

This study was performed in 62 legs from 31 Thai soft cadavers ( 9 male and 22 female) supported by the Chula Soft Cadaver Surgical Training Centre, Faculty of Medicine, Chulalongkorn University. The average age of the cadavers was $78.39 \pm 10.60$ years (age range 53-100). All cadaveric ankles and feet had no deformities, damage and history of previous surgery.

\section{Foot length measurement and surface marking}

The foot was aligned to neutral position by fixing with a supporting frame. The foot length was measured from the most posterior portion of the calcaneus to the end of the longest toe [30]. The line joining the medial end of plantar flexion crease at the base of the great toes $(\mathrm{MC})$ and the most prominent point of navicular tuberosity (NT) by palpation was created to be a reference line for locating the MKH surface landmark (Fig. 1A). 


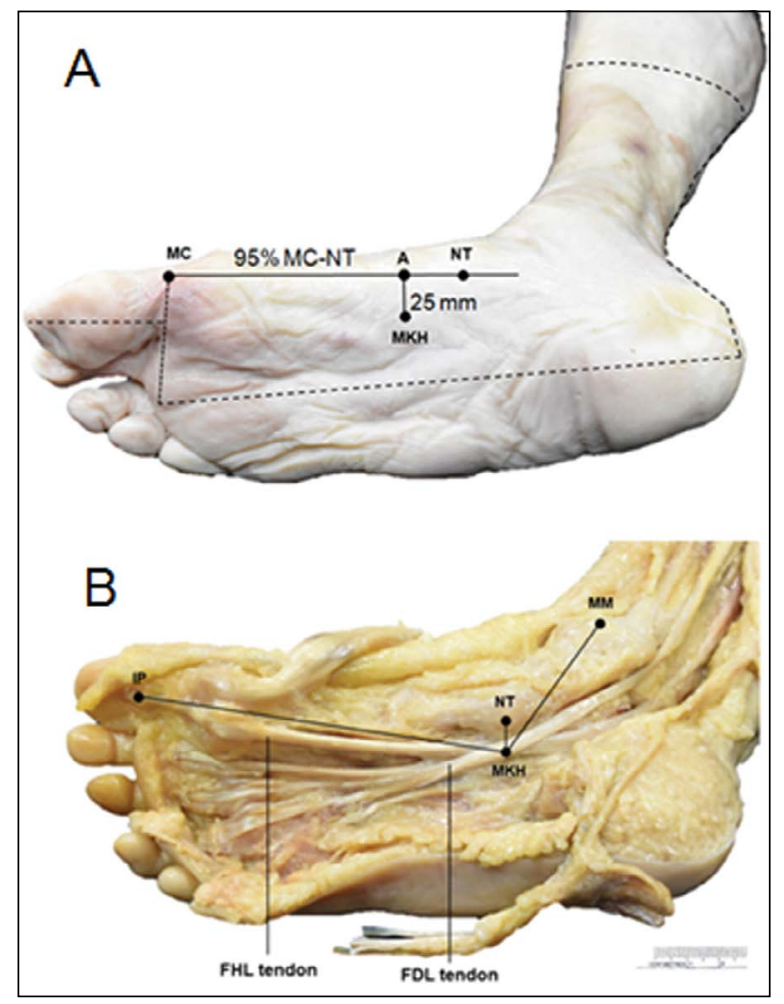

Figure 1. Plantar surface of right foot showing the skin incision and surface landmark of master knot of Henry (MKH); $\mathbf{A}$. Skin incision line (dot line), the line joining between the medial end of plantar flexion crease at the base of the great toes (MC) and the most prominent point of navicular tuberosity (NT) (MC-NT line), the surface location of MKH on MC-NT line; $\mathbf{B}$. The distance from MKH to the most prominent point of medial malleolus (MM), navicular tuberosity (NT) and first interphalangeal joint (IP); A - the perpendicular point of MKH on MC-NT line; FDL — flexor digitorum longus; $\mathrm{FHL}$ - flexor hallucis longus.

\section{Cadaveric dissection}

The skin incision along the dotted line as shown in Figure 1A was performed. The skin was dissected. Subcutaneous fatty tissue, flexor digitorum brevis and abductor hallucis muscle were removed to expose FHL tendon, FDL tendon and $\mathrm{MKH}$ in the plantar surface of foot (Fig. 1B). FHL was dissected further to its musculotendinous junction (MTJ) proximally and its insertion distally. The skin flab was turned down to cover the plantar surface of the foot and the location of MKH was marked on the skin surface. The perpendicular line from MKH to MC-NT line (MKH-A) was created (Fig. 1A). Point A might be located at NT, anterior to NT or posterior to NT on MC-NT line (Fig. 2). The most prominent point of medial malleolus (MM), sustentaculum tali (ST) and the midpoint of first interphalangeal joint of great toe (IP) were marked (Figs. 1B, 4A).

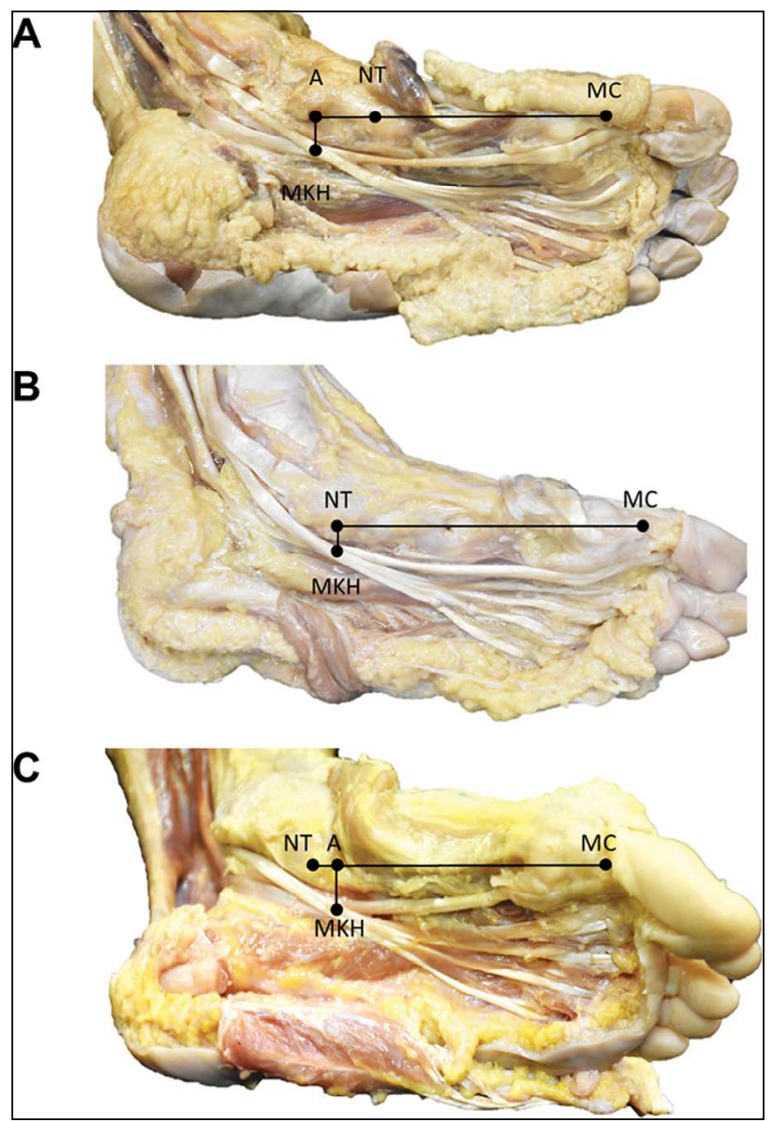

Figure 2. Plantar surface of left feet showing the surface location of master knot of Henry (MKH) on MC-NT line (point A); A. Posterior to NT; B. At NT; C. Anterior to NT; A - the perpendicular point of MKH on MC-NT line; MC - medial end of plantar flexor crease at the base of great toe; NT - navicular tuberosity.

\section{Observations and measurements}

Surface landmark and location of $\mathrm{MKH}$, its relationship to the neurovascular bundle. The surface landmark of MKH was determined by measuring the length of MC-NT, MKH-A and MC-A lines (Fig. 1A). The MC-A length was calculated into percentage of MC-NT length. To determine the location of $\mathrm{MKH}$ in the dissected specimen, the distances from $\mathrm{MKH}$ to MM, to NT and to IP was measured (Fig. 1B). The anatomical relationship between $\mathrm{MKH}$ and neurovascular bundle, including MPNVB and LPNVB were evaluated and the distance between their midpoints were recorded (Fig. 3). All distances were measured by standardised digital Vernier calliper (Mitutoyo ${ }^{\circledR}$ 0-150 mm; range $150 \mathrm{~mm}$, resolution $0.01 \mathrm{~mm}$ ).

In situ and ex vivo lengths of FHL tendon. To determine the in situ length of FHL tendon, the lengths from MTJ to ST, MTJ to MKH and MTJ to IP represent the length harvested through a single incision, dou- 


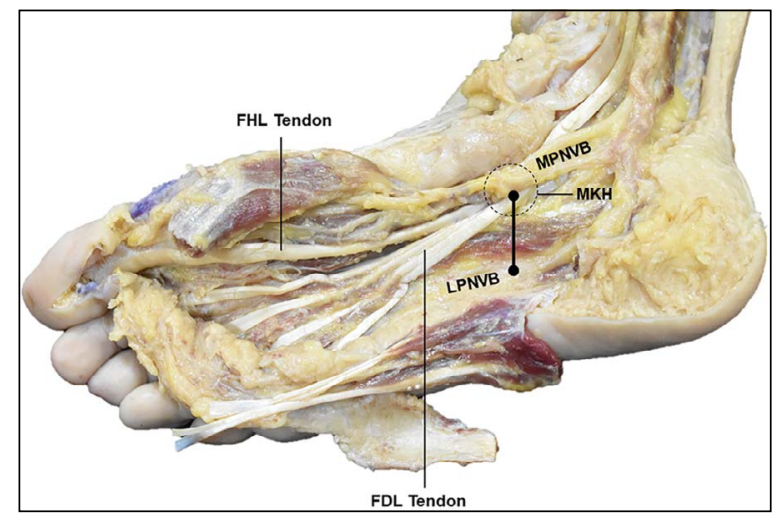

Figure 3. Plantar surface right foot showing the distance between master knot of Henry (MKH) and the midpoint of lateral plantar neurovascular bundle (LPNVB); MNVB - medial plantar neurovascular bundle; FDL — flexor digitorum longus; $\mathrm{FHL}$ — flexor hallucis longus.

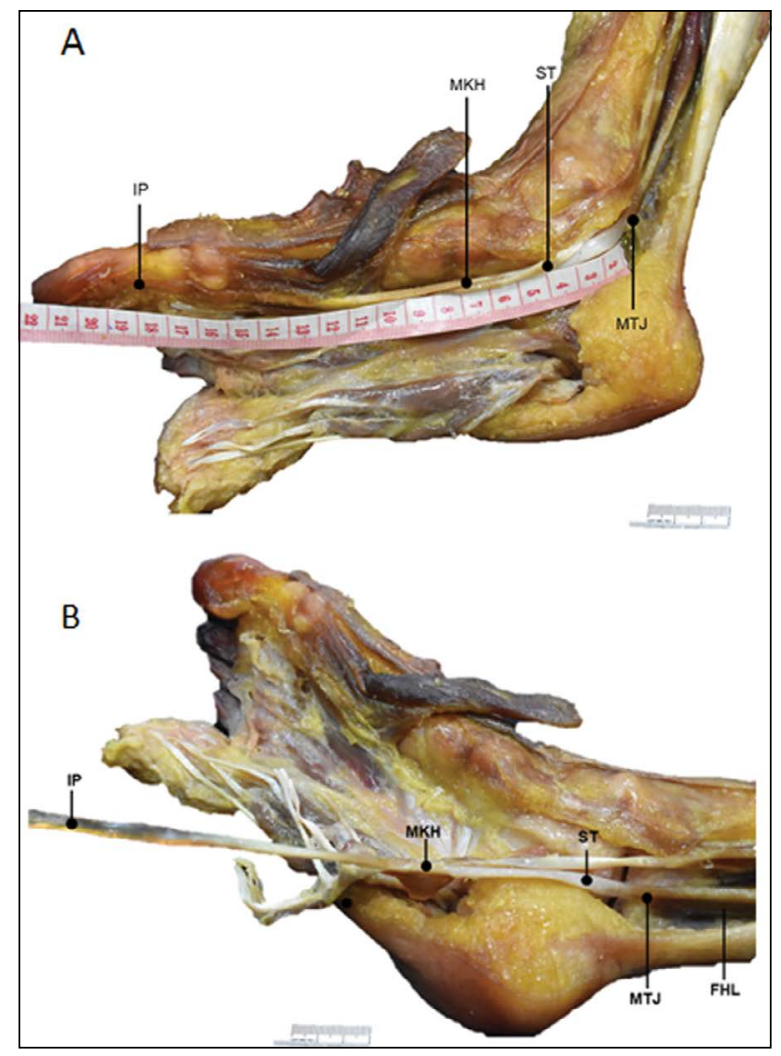

Figure 4. Plantar surface right foot showing the distances between musculotendinous junction (MTJ) of flexor hallucis longus (FHL) and first interphalangeal joint (IP), master knot of Henry (MKH), sustentaculum tali (ST); A. In situ tendon length; B. Ex vivo tendon length.

ble-incision and the minimally invasive technique, respectively (Fig. 4A).

To define the ex vivo length of FHL tendon, three points were marked on the FHL tendon at the level of ST,
MKH and IP. FHL tendon was cut at its insertion. Ex vivo length was measured from MTJ to those three points on $\mathrm{FHL}$ tendon by using a measuring tape (Butterfly ${ }^{\circledR}$ 0-150 cm; range $150 \mathrm{~cm}$, resolution $1 \mathrm{~mm}$ ) (Fig. 4B).

Each parameter was measured twice. The same digital Vernier calliper and measuring tape were used to ensure the consistency. All measurements were done by the same investigator.

\section{Statistical analysis}

Statistical analysis was performed by SPSS software version 22.0. All data from measurements were statistically analysed to demonstrate range, mean and standard deviation. To compare between genders, unpaired t-test (for parametric test) or Mann-Whitney $\mathrm{U}$ test (for nonparametric test) was used. The difference between in situ and ex vivo tendon length was examined with paired t-test (for parametric test) or Wilcoxon signed-rank test (for nonparametric test). A p-value of less than 0.05 was statistically significant. Pearson correlation test was used to assess the associative relationship between the foot length and the tendon length.

\section{Ethical consideration}

This cadaveric study has been approved by the Institutional Review Board (IRB) of the Faculty of Medicine, Chulalongkorn University (IRB No. 636/62).

\section{RESULTS}

\section{Foot length}

Results and analyses of the foot length are illustrated in Table 1. The mean length of foot in male and female was $246.50 \pm 12.02 \mathrm{~mm}$ and $224.64 \pm$ $\pm 11.63 \mathrm{~mm}$, respectively. A statistically significant difference was found between genders in both sides.

\section{Surface landmark and location of MKH}

Master knot of Henry location and surface landmark results and analyses are shown on Tables 2 and 3 . The location of MKH was identified at $117.11 \pm$ $\pm 1.00 \mathrm{~mm}$ proximal to $\mathrm{IP}, 26.28 \pm 4.75 \mathrm{~mm}$ under NT and $59.58 \pm 7.51 \mathrm{~mm}$ distal to MM. A statistically significant difference between genders was observed in MKH-IP of both sides and MKH-NT on right side.

Point A could be resided anterior to NT (66.1\%), at NT (27.4\%) and posterior to NT (6.5\%) (Table 3). The mean length of MC-NT and MC-A line were $107.36 \pm$ \pm 8.60 and $101.72 \pm 12.01 \mathrm{~mm}$, respectively. Point A was located at $94.75 \pm 8.43 \%$ of MC-NT line from 
Table 1. The mean foot length in male and female

\begin{tabular}{lcccc}
\hline Gender & \multicolumn{2}{c}{ Foot length $[\mathrm{mm}]$} & - mean \pm standard deviation (minimum-maximum) & P \\
\cline { 2 - 4 } & $\mathrm{Left}$ & Right & Total & 0.937 \\
\hline Male & $\mathrm{N}=9$ & $\mathrm{~N}=9$ & $\mathrm{~N}=18$ & \\
& $246.44 \pm 12.22(225.00-268.00)$ & $246.56 \pm 12.54(228.00-270.00)$ & $246.50 \pm 12.02(225.00-270.00)$ & \\
Female & $\mathrm{N}=22$ & $\mathrm{~N}=22$ & $\mathrm{~N}=44$ & \\
& $223.77 \pm 12.18(200.00-250.00)$ & $225.50 \pm 11.27(203.00-248.00)$ & $224.64 \pm 11.63(200.00-250.00)$ & - \\
Total & $\mathrm{N}=31$ & $\mathrm{~N}=31$ & $\mathrm{~N}=62$ & \\
& $230.35 \pm 15.91(20.00-26.80)$ & $231.61 \pm 15.01(203.00-270.00)$ & $230.98 \pm 15.35(200.00-270.00)$ & \\
$P$ & 0.00 & 0.00 & - & \\
\hline
\end{tabular}

Table 2. Location of MKH from IP, NT and MM, surface landmark of MKH, distances between MKH and NVB, location of MKH in term of percentage of the length of MC-NT line

\begin{tabular}{|c|c|c|c|c|c|c|c|}
\hline \multirow[t]{2}{*}{ Parameters } & \multicolumn{3}{|c|}{$\begin{array}{c}\text { Male — mean } \pm \text { standard deviation } \\
\text { (minimum-maximum) }\end{array}$} & \multicolumn{3}{|c|}{$\begin{array}{c}\text { Female — mean } \pm \text { standard deviation } \\
\text { (minimum-maximum) }\end{array}$} & \multirow[t]{2}{*}{ Total } \\
\hline & Left & Right & Total & Left & Right & Total & \\
\hline \multicolumn{8}{|c|}{ Location of MKH [mm]: } \\
\hline MKH-IP & $\begin{array}{c}124.69 \pm 9.04 \\
(106.56-137.88)\end{array}$ & $\begin{array}{r}126.39 \pm 12.39 \\
(110.24-151.27)\end{array}$ & $\begin{array}{c}125.54 \pm 10.55 \\
(106.56-151.27)\end{array}$ & $\begin{array}{c}113.30 \pm 8.11 \\
(93.14-129.50)\end{array}$ & $\begin{array}{c}114.03 \pm 6.95 \\
(100.27-128.20)\end{array}$ & $\begin{array}{c}113.66 \pm 7.47 \\
(93.14-129.50)\end{array}$ & $\begin{array}{r}117.11 \pm 1.00 \\
(93.14-151.27)\end{array}$ \\
\hline MKH-NT & $\begin{array}{l}29.45 \pm 7.06 \\
(21.38-40.91)\end{array}$ & $\begin{array}{l}27.88 \pm 4.15 \\
(19.53-33.16)\end{array}$ & $\begin{array}{l}28.67 \pm 5.68 \\
(19.53-40.91)\end{array}$ & $\begin{array}{l}26.21 \pm 3.51 \\
(17.93-33.73)\end{array}$ & $\begin{array}{l}24.40 \pm 4.32 \\
(18.30-35.87)\end{array}$ & $\begin{array}{l}25.31 \pm 3.99 \\
(17.93-35.87)\end{array}$ & $\begin{array}{l}26.28 \pm 4.75 \\
(17.93-40.91)\end{array}$ \\
\hline MKH-MM & $\begin{array}{l}63.32 \pm 9.80 \\
(47.02-78.71)\end{array}$ & $\begin{array}{l}60.02 \pm 11.02 \\
(34.59-75.27)\end{array}$ & $\begin{array}{l}61.67 \pm 10.26 \\
(34.59-78.71)\end{array}$ & $\begin{array}{l}60.36 \pm 6.49 \\
(48.91-78.07)\end{array}$ & $\begin{array}{l}57.10 \pm 5.06 \\
(44.43-67.05)\end{array}$ & $\begin{array}{l}58.72 \pm 5.98 \\
(44.43-78.07)\end{array}$ & $\begin{array}{l}59.58 \pm 7.51 \\
(34.59-78.71)\end{array}$ \\
\hline \multicolumn{8}{|c|}{ Surface landmark of MKH [mm]: } \\
\hline MC-NT & $\begin{array}{l}113.11 \pm 10.21 \\
(91.75-124.12)\end{array}$ & $\begin{array}{c}113.13 \pm 7.44 \\
(101.49-122.19)\end{array}$ & $\begin{array}{l}113.12 \pm 8.67 \\
(91.75-123.37)\end{array}$ & $\begin{array}{l}102.57 \pm 8.09 \\
(87.18-116.74)\end{array}$ & $\begin{array}{l}107.45 \pm 6.01 \\
(97.26-117.65)\end{array}$ & $\begin{array}{l}105.01 \pm 7.46 \\
(87.18-117.65)\end{array}$ & $\begin{array}{l}107.36 \pm 8.60 \\
(87.18-124.12)\end{array}$ \\
\hline MKH-A & $\begin{array}{l}29.10 \pm 8.83 \\
(19.39-41.85)\end{array}$ & $\begin{array}{l}27.89 \pm 4.86 \\
(25.11-35.53)\end{array}$ & $\begin{array}{l}28.49 \pm 6.94 \\
(19.39-41.85)\end{array}$ & $\begin{array}{l}24.18 \pm 3.33 \\
(17.26-32.27)\end{array}$ & $\begin{array}{r}23.26 \pm 4.39 \\
(16.89-34.15)\end{array}$ & $\begin{array}{l}23.72 \pm 3.88 \\
(16.89-34.15)\end{array}$ & $\begin{array}{l}25.11 \pm 5.37 \\
(16.89-41.85)\end{array}$ \\
\hline MC-A & $\begin{array}{l}111.59 \pm 11.01 \\
(90.62-126.71)\end{array}$ & $\begin{array}{l}110.87 \pm 15.39 \\
(87.26-139.77)\end{array}$ & $\begin{array}{l}111.23 \pm 12.99 \\
(87.26-139.77)\end{array}$ & $\begin{array}{l}95.92 \pm 10.55 \\
(76.05-116.74)\end{array}$ & $\begin{array}{c}99.75 \pm 7.40 \\
(86.91-115.63)\end{array}$ & $\begin{array}{c}97.83 \pm 9.21 \\
(76.05-116.74)\end{array}$ & $\begin{array}{l}101.72 \pm 12.01 \\
(76.05-139.77)\end{array}$ \\
\hline \multicolumn{8}{|c|}{ Distance between MKH and NVB [mm]: } \\
\hline MKH-LPNVB & $\begin{array}{l}17.98 \pm 6.09 \\
(9.59-30.29)\end{array}$ & $\begin{array}{r}19.91 \pm 5.32 \\
(13.05-28.85)\end{array}$ & $\begin{array}{l}18.94 \pm 5.64 \\
(9.59-30.29)\end{array}$ & $\begin{array}{l}15.17 \pm 3.84 \\
(8.44-22.05)\end{array}$ & $\begin{array}{l}17.61 \pm 3.56 \\
(7.11-24.38)\end{array}$ & $\begin{array}{l}16.39 \pm 3.86 \\
(7.11-24.38)\end{array}$ & $\begin{array}{l}17.13 \pm 3.55 \\
(7.11-30.29)\end{array}$ \\
\hline MKH-MPNVB & 0 & 0 & 0 & 0 & 0 & 0 & 0 \\
\hline $\begin{array}{l}\text { Location of MKH } \\
\text { in term of per- } \\
\text { centage of MC-NT } \\
\text { length }(\%)\end{array}$ & $\begin{array}{c}98.84 \pm 7.77 \\
(87.77-110.28)\end{array}$ & $\begin{array}{r}98.34 \pm 15.90 \\
(82.21-137.72)\end{array}$ & $\begin{array}{c}98.56 \pm 11.98 \\
(82.21-137.72)\end{array}$ & $\begin{array}{c}93.47 \pm 6.36 \\
(75.83-107.16)\end{array}$ & $\begin{array}{c}92.89 \pm 5.65 \\
(79.82-100.00)\end{array}$ & $\begin{array}{c}93.18 \pm 5.95 \\
(75.83-107.16)\end{array}$ & $\begin{array}{c}94.75 \pm 8.43 \\
(75.83-137.72)\end{array}$ \\
\hline
\end{tabular}

A — perpendicular point of MKH on MC-NT line; IP — first interphalangeal joint of great toe; LPNVB — lateral plantar neurovascular bundles; MC — medial end of plantar flexion crease at the base of great toes; MKH — master knot of Henry; MM — medial malleolus; MPNVB — medial plantar neurovascular bundles; NT — navicular tuberosity

$\mathrm{MC}$ (Table 2). The mean perpendicular length from MKH to A (MKH-A) was $25.11 \pm 5.37 \mathrm{~mm}$ (Table 2). A statistically significant difference between genders was present in MC-A on both sides and MKH-A on right side.

\section{Relationship between MKH and plantar neurovascular bundle}

The MPNVB lied very closely to MKH in all cases; therefore, no distance could be measured. In contrast, a mean distance of $17.13 \pm 3.55 \mathrm{~mm}$ was observed between LPNVB and MKH without a statistically significant difference between genders (Table 2).

\section{Length of FHL tendon}

The mean in situ and ex vivo length of FHL tendon graft, harvested by three different incision techniques, are shown on Table 4. A statistically significant difference was found between genders in MTJ-IP of in situ and ex vivo length of both sides $(p<0.05)$. The 
Table 3. Prevalence of master knot of Henry (MKH) location on MC-NT line

\begin{tabular}{|c|c|c|c|c|c|c|c|}
\hline \multirow[t]{2}{*}{ Location of MKH } & \multicolumn{3}{|c|}{ Male } & \multicolumn{3}{|c|}{ Female } & \multirow{2}{*}{$\begin{array}{c}\text { Total } \\
(\mathrm{n}=62)\end{array}$} \\
\hline & Left $(n=9)$ & Right $(n=9)$ & Total $(n=18)$ & Left $(n=22)$ & Right $(n=22)$ & Total $(n=44)$ & \\
\hline At NT & $4(44.4 \%)$ & $3(33.3 \%)$ & $7(38.9 \%)$ & $4(18.2 \%)$ & $6(27.3 \%)$ & $10(22.7 \%)$ & $17(27.4 \%)$ \\
\hline Anterior to NT & $3(33.33 \%)$ & $5(55.6 \%)$ & $8(44.4 \%)$ & 17 (77.3\%) & $16(72.7 \%)$ & $33(75.0 \%)$ & $41(66.1 \%)$ \\
\hline Posterior to NT & $2(22.22 \%)$ & $1(11.1 \%)$ & $3(16.7 \%)$ & $1(4.5 \%)$ & $0(0 \%)$ & $1(2.3 \%)$ & $4(6.5 \%)$ \\
\hline
\end{tabular}

MC — medial end of plantar flexion crease at the base of great toes; MM — medial malleolus; NT — navicular tuberosity

Table 4. In situ and ex vivo length of harvested flexor hallucis longus tendon from single incision (MTJ-ST), double incision (MTJ-MKH) and minimally invasive techniques (MTJ-IP)

\begin{tabular}{|c|c|c|c|c|c|c|c|}
\hline \multirow[t]{3}{*}{ Techniques } & \multicolumn{6}{|c|}{ Genders } & \multirow[t]{3}{*}{ Total $(n=62)$} \\
\hline & \multicolumn{3}{|c|}{$\begin{array}{c}\text { Male - mean } \pm \text { standard deviation } \\
\text { (minimum-maximum) }\end{array}$} & \multicolumn{3}{|c|}{$\begin{array}{l}\text { Female - mean } \pm \text { standard deviation } \\
\text { (minimum-maximum) }\end{array}$} & \\
\hline & Left $(n=9)$ & Right $(n=9)$ & Total $(n=18)$ & Left ( $n=22)$ & Right (n = 22) & Total $(n=44)$ & \\
\hline \multicolumn{8}{|l|}{ In situ length: } \\
\hline MTJ-ST & $\begin{array}{l}43.22 \pm 12.14 \\
(21.00-60.00)\end{array}$ & $\begin{array}{l}41.56 \pm 8.35 \\
(30.00-55.00)\end{array}$ & $\begin{array}{l}42.39 \pm 10.15 \\
(21.00-60.00)\end{array}$ & $\begin{array}{l}37.50 \pm 10.15 \\
(15.00-62.00)\end{array}$ & $\begin{array}{l}37.86 \pm 12.01 \\
(19.00-71.00)\end{array}$ & $\begin{array}{l}37.68 \pm 11.00 \\
(15.00-71.00)\end{array}$ & $\begin{array}{l}39.05 \pm 10.88 \\
(15.00-71.00)\end{array}$ \\
\hline MTJ-MKH & $\begin{array}{l}76.78 \pm 9.55 \\
(67.00-95.00)\end{array}$ & $\begin{array}{l}75.78 \pm 10.19 \\
(60.00-91.00)\end{array}$ & $\begin{array}{l}76.28 \pm 9.60 \\
(60.00-95.00)\end{array}$ & $\begin{array}{l}71.00 \pm 8.09 \\
(59.00-92.00)\end{array}$ & $\begin{array}{l}73.59 \pm 11.51 \\
(54.00-95.00)\end{array}$ & $\begin{array}{l}72.29 \pm 9.92 \\
(54.00-95.00)\end{array}$ & $\begin{array}{l}73.45 \pm 9.91 \\
(54.00-95.00)\end{array}$ \\
\hline MTJ-IP & $\begin{array}{l}209.44 \pm 17.67 \\
(181.00-240.00)\end{array}$ & $\begin{array}{l}209.56 \pm 15.32 \\
(188.00-240.00)\end{array}$ & $\begin{array}{l}209.50 \pm 16.00 \\
(181.00-240.00)\end{array}$ & $\begin{array}{c}192.22 \pm 9.12 \\
(174.00-215.00)\end{array}$ & $\begin{array}{r}194.32 \pm 10.33 \\
(179.00-215.00)\end{array}$ & $\begin{array}{c}193.27 \pm 9.68 \\
(174.00-215.00)\end{array}$ & $\begin{array}{r}197.98 \pm 13.89 \\
(174.00-240.00)\end{array}$ \\
\hline \multicolumn{8}{|l|}{ Ex vivo length: } \\
\hline MTJ-ST & $\begin{array}{l}38.33 \pm 10.22 \\
(19.00-50.00)\end{array}$ & $\begin{array}{l}36.67 \pm 7.36 \\
(25.00-46.00)\end{array}$ & $\begin{array}{l}37.50 \pm 8.68 \\
(19.00-50.00)\end{array}$ & $\begin{array}{c}33.00 \pm 10.64 \\
(11.00-57.00)\end{array}$ & $\begin{array}{l}33.36 \pm 10.88 \\
(15.00-58.00)\end{array}$ & $\begin{array}{l}33.18 \pm 10.63 \\
(11.00-58.00)\end{array}$ & $\begin{array}{l}34.43 \pm 10.23 \\
(11.00-58.00)\end{array}$ \\
\hline MTJ-MKH & $\begin{array}{l}71.67 \pm 8.90 \\
(64.00-90.00)\end{array}$ & $\begin{array}{l}70.44 \pm 9.36 \\
(57.00-87.00)\end{array}$ & $\begin{array}{l}71.06 \pm 8.88 \\
(57.00-90.00)\end{array}$ & $\begin{array}{l}66.45 \pm 8.73 \\
(54.00-89.00)\end{array}$ & $\begin{array}{l}68.82 \pm 10.40 \\
(51.00-84.00)\end{array}$ & $\begin{array}{l}67.64 \pm 9.56 \\
(51.00-89.00)\end{array}$ & $\begin{array}{l}68.63 \pm 9.43 \\
(51.00-90.00)\end{array}$ \\
\hline MTJ-IP & $\begin{array}{l}203.56 \pm 18.77 \\
(174.00-236.00)\end{array}$ & $\begin{array}{l}203.78 \pm 14.94 \\
(184.00-235.00)\end{array}$ & $\begin{array}{l}203.67 \pm 16.46 \\
(174.00-236.00)\end{array}$ & $\begin{array}{c}186.36 \pm 9.21 \\
(167.00-209.00)\end{array}$ & $\begin{array}{c}187.50 \pm 9.69 \\
(173.00-205.00)\end{array}$ & $\begin{array}{c}186.93 \pm 9.36 \\
(167.00-209.00)\end{array}$ & $\begin{array}{r}191.79 \pm 14.00 \\
(167.00-236.00)\end{array}$ \\
\hline
\end{tabular}

IP — first interphalangeal joint of great toe; MKH — master knot of Henry; MTJ — musculotendinous junction; NT — navicular tuberosity; ST — sustentaculum tali

Table 5. Length of harvested flexor hallucis longus tendon in term of percentage of the foot length

\begin{tabular}{|c|c|c|c|c|c|c|c|}
\hline \multirow[t]{3}{*}{ Techniques } & \multicolumn{6}{|c|}{ Genders } & \multirow[t]{3}{*}{ Total $(n=62)$} \\
\hline & \multicolumn{3}{|c|}{$\begin{array}{l}\text { Male - mean } \pm \text { standard deviation } \\
\text { (minimum-maximum) }\end{array}$} & \multicolumn{3}{|c|}{$\begin{array}{l}\text { Female - mean } \pm \text { standard deviation } \\
\text { (minimum-maximum) }\end{array}$} & \\
\hline & Left $(n=9)$ & Right $(n=9)$ & Total $(n=18)$ & Left $(n=22)$ & Right ( $n=22$ ) & Total $(n=44)$ & \\
\hline \multicolumn{8}{|l|}{ In situ length: } \\
\hline MTJ-ST & $\begin{array}{l}17.45 \pm 4.64 \\
(9.33-24.29)\end{array}$ & $\begin{array}{l}16.84 \pm 3.27 \\
(12.24-22.82)\end{array}$ & $\begin{array}{l}17.14 \pm 3.90 \\
(9.33-24.29)\end{array}$ & $\begin{array}{l}16.84 \pm 4.72 \\
(6.38-28.84)\end{array}$ & $\begin{array}{l}16.92 \pm 5.77 \\
(8.19-33.81)\end{array}$ & $\begin{array}{l}16.88 \pm 5.21 \\
(6.38-33.81)\end{array}$ & $\begin{array}{l}16.96 \pm 4.84 \\
(6.38-33.81)\end{array}$ \\
\hline MTJ-MKH & $\begin{array}{l}31.18 \pm 3.69 \\
(27.31-35.56)\end{array}$ & $\begin{array}{l}30.76 \pm 2.91 \\
(26.15-36.93)\end{array}$ & $\begin{array}{l}30.97 \pm 3.79 \\
(26.15-36.93)\end{array}$ & $\begin{array}{l}31.81 \pm 3.89 \\
(25.11-39.07)\end{array}$ & $\begin{array}{l}32.70 \pm 5.36 \\
(23.40-45.24)\end{array}$ & $\begin{array}{l}32.26 \pm 4.65 \\
(23.40-45.24)\end{array}$ & $\begin{array}{l}31.88 \pm 4.43 \\
(23.40-45.24)\end{array}$ \\
\hline MTJ-IP & $\begin{array}{r}84.89 \pm 3.89 \\
(74.58-88.06)\end{array}$ & $\begin{array}{l}84.97 \pm 3.90 \\
(80.77-93.36)\end{array}$ & $\begin{array}{l}84.93 \pm 3.78 \\
(78.75-93.36)\end{array}$ & $\begin{array}{l}86.02 \pm 3.91 \\
(75.74-92.09)\end{array}$ & $\begin{array}{l}85.83 \pm 3.51 \\
(79.91-93.75)\end{array}$ & $\begin{array}{l}85.92 \pm 3.67 \\
(75.74-93.75)\end{array}$ & $\begin{array}{r}85.63 \pm 3.70 \\
(75.74-93.75)\end{array}$ \\
\hline \multicolumn{8}{|c|}{ Ex vivo length: } \\
\hline MTJ-ST & $\begin{array}{l}15.47 \pm 3.85 \\
(8.44-20.24)\end{array}$ & $\begin{array}{l}14.87 \pm 2.91 \\
(12.24-18.67)\end{array}$ & $\begin{array}{l}15.17 \pm 3.33 \\
(8.44-20.24)\end{array}$ & $\begin{array}{l}14.83 \pm 4.91 \\
(4.78-26.51)\end{array}$ & $\begin{array}{l}14.91 \pm 5.15 \\
(6.47-27.62)\end{array}$ & $\begin{array}{l}14.87 \pm 4.97 \\
(4.78-27.62)\end{array}$ & $\begin{array}{l}14.96 \pm 4.53 \\
(4.78-27.62)\end{array}$ \\
\hline MTJ-MKH & $\begin{array}{l}29.09 \pm 3.31 \\
(25.77-33.58)\end{array}$ & $\begin{array}{l}28.59 \pm 3.70 \\
(24.23-34.80)\end{array}$ & $\begin{array}{l}28.84 \pm 3.41 \\
(24.23-34.80)\end{array}$ & $\begin{array}{l}29.77 \pm 4.07 \\
(23.20-37.21)\end{array}$ & $\begin{array}{l}30.57 \pm 4.78 \\
(23.18-40.00)\end{array}$ & $\begin{array}{l}32.26 \pm 4.65 \\
(23.18-40.00)\end{array}$ & $\begin{array}{l}29.79 \pm 4.16 \\
(23.18-40.00)\end{array}$ \\
\hline MTJ-IP & $\begin{array}{l}82.48 \pm 4.49 \\
(74.58-88.06)\end{array}$ & $\begin{array}{l}82.61 \pm 3.34 \\
(79.62-89.21)\end{array}$ & $\begin{array}{l}82.55 \pm 3.84 \\
(74.58-89.21)\end{array}$ & $\begin{array}{c}83.38 \pm 3.71 \\
(74.89-90.23)\end{array}$ & $\begin{array}{l}83.38 \pm 3.71 \\
(74.89-90.23)\end{array}$ & $\begin{array}{c}83.31 \pm 3.95 \\
(74.89-94.76)\end{array}$ & $\begin{array}{c}83.09 \pm 3.90 \\
(74.58-94.76)\end{array}$ \\
\hline
\end{tabular}

IP — first interphalangeal joint of great toe; MKH — master knot of Henry; MTJ — musculotendinous junction; NT — navicular tuberosity; ST — sustentaculum tali 
mean length of tendon between in situ and ex vivo was significantly different in all techniques $(p<0.05)$. Moreover, a moderate positive correlation between foot length and tendon length was found in MTJ-IP of both in situ and ex vivo tendon length $(r=0.52$ and 0.56 , respectively).

The length of FHL tendon was calculated in term of percentage of foot length as shown in Table 5. In situ and ex vivo tendon lengths were $16.96 \pm 4.84$ and $14.96 \pm 4.53 \%, 31.88 \pm 4.43$ and $29.79 \pm 4.16 \%$, $85.63 \pm 3.70$ and $83.09 \pm 3.90 \%$ of foot length in single incision, double incision, and minimally invasive techniques, respectively.

\section{DISCUSSION}

Achilles tendinopathy is a painful condition that can occur in both active and inactive people [11]. Despite of noninvasive treatments such as physical therapy, orthotics and drugs, surgical intervention might be necessary when clinical outcome remained disappointing $[6,12]$. FHL tendon is a common tendon used in the augmentation of the Achilles tendon because it is easy to harvest and provides good to excellent functional outcomes and pain relief regardless of the technique used to harvest the tendon $[4,6,29]$.

The shape and morphology of the foot vary among ethnicities, genders, and individuals [10, 15, 21, 24, 31]. Foot length was used in this study to anticipate anatomical data which is significant for FHL tendon transfer. Asian foot length is shorter than that of North American and European. In Asians, the most frequent length was $255 \mathrm{~mm}$ for male and $235 \mathrm{~mm}$ for female [9]. In this study, the mean foot lengths were $246 \mathrm{~mm}$ and $225 \mathrm{~mm}$ in male and female respectively. A significant difference between genders was found similar to the previous reports [3,22].

Master knot of Henry has been widely utilized as a surgical landmark for the FHL tendon graft harvesting especially in double incision technique [2]. The first IP joint and NT were used to localize MKH by Mao et al. [13], in Asian embalmed cadavers. Moreover, Beger et al. [2] and Vasudha et al. [28] further investigated the precise location of the MKH from MM, NT and first IP joint in Turkish and Indian formalin fixed cadavers, respectively. According to the results of this study, the location of MKH resided proximal to the first IP joint, inferior to NT and distal to $M M$ which resembled findings of previous reports (Table 6) $[2,28]$.
Although there were several reports about the location of the MKH, they did not take surface landmarks for localizing MKH into account. Medial end of plantar flexion crease at the base of great toe (MC) and navicular tuberosity (NT), which could be clearly identified and palpated, were used to determine the surface localization of MKH in this study. For accuracy and easy application in clinical practice, MC-NT line and A which is the perpendicular point of MKH on MC-NT line were defined. Approximately, MKH located at $95 \%$ of MC-NT line from MC with a perpendicular distance of $25 \mathrm{~mm}$ from MC-NT line. However, our results revealed that point $A$ could be located anterior, posterior and at the NT on MC-NT line. Nevertheless, MKH was located posterior to NT in only $6.5 \%$ of cases.

Medial and lateral plantar nerves (MPN and LPN) are the branches of posterior tibial nerve which supply skin and intrinsic muscle of the sole. Anatomically, MPN travels along the plantar surface of FDL tendon and passes through MKH [16]. LPN passes obliquely between flexor digitorum brevis and quadratus plantae to the lateral side of the foot. The anatomical relationship between plantar nerves and MKH was reported by Mao et al. [14] in embalmed cadavers. They found a mean distance of $5.26 \mathrm{~mm}$ between MPN and MKH, and $15.50 \mathrm{~mm}$ between LPN and MKH which was different from the result of this study. In all specimens of this study, there was no distance between MPNVB and MKH and a longer distance of $17.13 \pm 3.55 \mathrm{~mm}$ was observed between LPNVB and MKH. This might be due to the different methods of cadaveric fixation. In embalmed cadaver, most tissues are rigid and joints cannot be moved freely which may affect the location of anatomical structures [23]. The proximity of MKH and MPNVB might lead to neurovascular bundle injury. The injuries of the distal branches of the posterior tibial nerve and artery were reported previously $[7,13,14,18]$. The transection of tendon that was performed near MKH in double incision technique may cause MPN or LPN injury [13]. In the literature, it was hypothesized that difficult harvesting might be the cause of nerve injury [16]. Nerve injury might be partial but not significant enough to cause clinical symptoms and long periods of casting after surgery could prohibit the detection of symptoms [16]. Nevertheless, caution is required to preserve this neurovascular bundle especially when distal transection is performed blindly $[25,27]$. More- 
Table 6. Comparison of the distances from master knot of Henry (MKH) to anatomical landmarks and in situ flexor hallucis longus tendon length

\begin{tabular}{|c|c|c|c|c|c|}
\hline & This study, 2020 & Vasudha et al., 2019 [28] & Beger et al., 2018 [2] & Mao et al., 2015 [13] & Tashjian et al., 2003 [27] \\
\hline Ethnic & Thai & Indian & Turkish & Asian & United States \\
\hline Cadaveric type & Soft & Formalin fixed & Formalin fixed & Embalmed & Fresh frozen \\
\hline Number of specimen & 62 & $L: 36 ; R: 36$ & 20 & 64 & 14 \\
\hline \multicolumn{6}{|c|}{ Distances from MKH to landmarks [cm]: } \\
\hline MM & $\begin{array}{l}5.96 \pm 0.75 \\
(3.46-7.87)\end{array}$ & $\begin{array}{c}\text { L: } 6.07 \pm 1.25 \\
(4.03-9.00) \\
\text { R: } 6.10 \pm 1.17 \\
(4.26-8.50)\end{array}$ & $\begin{array}{l}5.93 \pm 0.74 \\
(4.72-7.35)\end{array}$ & - & - \\
\hline NT & $\begin{array}{l}2.63 \pm 0.48 \\
(1.79-4.09)\end{array}$ & $\begin{array}{c}\mathrm{L}: 2.99 \pm 0.96 \\
(1.50-5.50) \\
\mathrm{R}: 3.24 \pm 0.93 \\
(1.64-5.00)\end{array}$ & $\begin{array}{l}1.75 \pm 0.39 \\
(1.11-2.44)\end{array}$ & $\begin{array}{l}2.21 \pm 0.34 \\
(1.59-3.04)\end{array}$ & - \\
\hline $\mathbb{P}$ & $\begin{array}{l}11.71 \pm 1.00 \\
(9.31-15.13)\end{array}$ & $\begin{array}{c}\text { L: } 11.97 \pm 1.11 \\
(9.32-14.2) \\
\text { R: } 12.50 \pm 0.89 \\
(9.77-14.46)\end{array}$ & $\begin{array}{c}12.61 \pm 1.11 \\
(10.33-14.09)\end{array}$ & $\begin{array}{l}10.89 \pm 1.08 \\
(13.04-9.22)\end{array}$ & - \\
\hline \multicolumn{6}{|l|}{ Tendon length [cm]: } \\
\hline $\begin{array}{l}\text { Single incision } \\
\text { technique }\end{array}$ & $\begin{array}{l}3.90 \pm 1.09 \\
(1.50-7.10)\end{array}$ & - & $\begin{array}{l}5.75 \pm 0.63 \\
(4.52-6.86)\end{array}$ & $\begin{array}{l}5.08 \pm 1.09 \\
(3.32-10.35)\end{array}$ & $\begin{array}{c}5.16 \pm 1.29 \\
(3.4-6.9)\end{array}$ \\
\hline $\begin{array}{l}\text { Double incision } \\
\text { technique }\end{array}$ & $\begin{array}{l}7.34 \pm 0.99 \\
(5.40-9.50)\end{array}$ & - & $\begin{array}{l}7.03 \pm 0.86 \\
(5.77-8.80)\end{array}$ & $\begin{array}{c}6.72 \pm 1.02 \\
(4.69-12.09)\end{array}$ & $\begin{array}{c}8.09 \pm 1.63 \\
(5.1-11.1)\end{array}$ \\
\hline $\begin{array}{l}\text { Minimally invasive } \\
\text { technique }\end{array}$ & $\begin{array}{c}19.80 \pm 1.39 \\
(17.40-24.00)\end{array}$ & - & $\begin{array}{l}20.22 \pm 1.32 \\
(16.82-21.97)\end{array}$ & $\begin{array}{r}17.49 \pm 1.80 \\
(13.51-20.52)\end{array}$ & - \\
\hline
\end{tabular}

IP — first interphalangeal joint of great toe; L — left; MM — medial malleolus; NT — navicular tuberosity; R — right

over, tendon disease in the region of MKH may lead to the entrapment of MPN [5].

In this study, the length of tendon graft with three different incision techniques (single incision, double incision and minimally invasive technique) was quantified. Previous researches reported that the in situ length of harvested FHL tendon were different between techniques (Table 6) $[2,13,27]$. The length of tendon graft from single incision technique in this study was shorter than previous studies. In double incision technique, our result was longer when compared to those of Mao et al. [13] and Beger et al. [2], but shorter than that of Tashjian et al. [27]. Furthermore, the length of tendon graft from minimally invasive technique was found to be longer when compares with Mao et al. [13]. Nevertheless, it was shorter than the mean length from Beger et al. [2]. These differences might be caused by the different ethnic backgrounds, cadaveric preservation technique and position of foot and ankle during measurement.

Ex vivo length of tendon graft has never been reported previously. Ex vivo length refers to the length of tendon after it is cut from the insertion point, which may be more similar to the length of harvested tendon for transfer. Our results revealed significant differences between in situ and ex vivo length of tendon from all techniques. Ex vivo tendon length was shorter than in situ tendon length by about $4.5 \mathrm{~mm}$ in single incision and double incision techniques and $6.0 \mathrm{~mm}$ in minimally invasive technique. The shorter tendon might result from loss of tension after it was cut from the insertion site in the foot. The correlation between tendon length and foot length was analysed for clinical benefit. Our results showed a moderate positive correlation between them. The lengths of harvested tendon from single incision, double incision and minimally invasive technique were about $15 \%$, $30 \%$, and $85 \%$ of foot length, respectively. Thus, it might be possible to estimate the length of harvested tendon from the foot length.

This study offers some benefits as it identifies the precise surface location of MKH which will make it easier to identify the incision site and improve the clinical efficacy of the surgery. Understanding the relation between $\mathrm{MKH}$ and neurovascular bundle can assist the clinician to avoid iatrogenic injury. The in 
situ and ex vivo length of FHL tendon could guide surgeons to designs personalized operation techniques that are appropriate for each patient. Therefore, the knowledge of this investigation can enhance the clinical efficacy of foot and ankle surgery and help minimize potential complications.

\section{CONCLUSIONS}

Master knot of Henry resides distal to MM, under NT and proximal to IP with MPNVB residing closely to MKH. Surface localisation of MKH can be located at $95 \%$ of MC-NT line from MC with a perpendicular distance of $25 \mathrm{~mm}$ from MC-NT line. The ex vivo lengths of tendon graft in all techniques were significantly shorter than in situ length. Foot length, MKH-IP, MKH-NT, MC-A, MKH-A and the lengths of FHL tendon graft from minimally invasive technique had statistically significant differences between genders.

\section{Acknowledgements}

This study was supported by the $100^{\text {th }}$ Anniversary Chulalongkorn University Fund for Doctoral Scholarship. The authors would like to thank and offer sincere gratitude to all those who have donated their bodies for research. Authors would like to thank Dr. Jiran Apinun from Department of Orthopaedics for providing clinical information. Special thanks are extended to the technical staffs of the Chula Soft Cadaver Surgical Training Centre, Department of Anatomy, Faculty of Medicine, Chulalongkorn University for their support in cadaveric care.

\section{REFERENCES}

1. Alhaug OK, Berdal G, Husebye EE, et al. Flexor hallucis longus tendon transfer for chronic Achilles tendon rupture. A retrospective study. Foot Ankle Surg. 2019; 25(5): 630-635, doi: 10.1016/j.fas.2018.07.002, indexed in Pubmed: 30321934.

2. Beger O, Elvan Ö, Keskinbora M, et al. Anatomy of Master Knot of Henry: A morphometric study on cadavers. Acta Orthop Traumatol Turc. 2018; 52(2): 134-142, doi: 10.1016/j.aott.2018.01.001, indexed in Pubmed: 29366540.

3. Chaiwanichsiri $D$, Tantisiriwat $N$, Janchai S. Proper shoe sizes for Thai elderly. Foot (Edinb). 2008; 18(4): 186-191, doi: 10.1016/j.foot.2008.05.001, indexed in Pubmed: 20307435.

4. Coughlin MJ, Saltzman CL, Anderson RB. Mann's surgery of the foot and ankle. 9th ed. Elsevier Inc., Philadelphia 2014: 1617-1620.

5. Donovan A, Rosenberg ZS, Bencardino JT, et al. Plantar tendons of the foot: MR imaging and US. Radiographics. 2013; 33(7): 2065-2085, doi: 10.1148/rg.337125167, indexed in Pubmed: 24224599.
6. Hahn F, Meyer P, Maiwald C, et al. Treatment of chronic achilles tendinopathy and ruptures with flexor hallucis tendon transfer: clinical outcome and MRI findings. Foot Ankle Int. 2008; 29(8): 794-802, doi: 10.3113/ FAl.2008.0794, indexed in Pubmed: 18752777.

7. Herbst SA, Miller SD. Transection of the medial plantar nerve and hallux cock-up deformity after flexor hallucis longus tendon transfer for Achilles tendinitis: case report. Foot Ankle Int. 2006; 27(8): 639-641, doi: 10.1177/107110070602700814, indexed in Pubmed: 16919220 .

8. Hockenbury R, Sammarco G. Medial sliding calcaneal osteotomy with flexor hallucis longus transfer for the treatment of posterior tibial tendon insufficiency. Foot and Ankle Clinics. 2001; 6(3): 569-581, doi: 10.1016/ s1083-7515(03)00114-1.

9. Jurca A, Žabkar J, Džeroski S. Analysis of 1.2 million foot scans from North America, Europe and Asia. Sci Rep. 2019; 9(1): 19155, doi: 10.1038/s41598-019-55432-z, indexed in Pubmed: 31844106.

10. Kouchi M. Foot dimensions and foot shape: differences due to growth, generation and ethnic origin. Anthropol Sci. 1998; 106(Suppl.): 161-188, doi: 10.1537/ase.106. supplement_161.

11. Lake JE, Ishikawa SN. Conservative treatment of Achilles tendinopathy: emerging techniques. Foot Ankle Clin. 2009; 14(4): 663-674, doi: 10.1016/j.fcl.2009.07.003, indexed in Pubmed: 19857840.

12. Lopez RG, Jung HG. Achilles tendinosis: treatment options. Clin Orthop Surg. 2015; 7(1): 1-7, doi: 10.4055/ cios.2015.7.1.1, indexed in Pubmed: 25729512.

13. Mao H, Shi Z, Wapner KL, et al. Anatomical study for flexor hallucis longus tendon transfer in treatment of Achilles tendinopathy. Surg Radiol Anat. 2015; 37(6): 639-647, doi: 10.1007/s00276-014-1399-y, indexed in Pubmed: 25542244

14. Mao H, Dong W, Shi Z, et al. Anatomical study of the neurovascular in flexor hallucis longus tendon transfers. Sci Rep. 2017; 7(1): 14202, doi: 10.1038/s41598-01713742-0, indexed in Pubmed: 29079740.

15. Mauch M, Grau S, Krauss I, et al. A new approach to children's footwear based on foot type classification. Ergonomics. 2009; 52(8): 999-1008, doi: 10.1080/00140130902803549, indexed in Pubmed: 19629814

16. Mulier T, Rummens E, Dereymaeker G. Risk of neurovascular injuries in flexor hallucis longus tendon transfers: an anatomic cadaver study. Foot Ankle Int. 2007; 28(8): 910-915, doi: 10.3113/FAl.2007.0910, indexed in Pubmed: 17697656.

17. Murphy RL, Womack JW, Anderson T. Technique tip: a new technique for harvest of the flexor hallucis longus tendon. Foot Ankle Int. 2010; 31(5): 457-459, doi: 10.3113/ FAl.2010.0457, indexed in Pubmed: 20460076.

18. Myerson MS, Corrigan J. Treatment of posterior tibial tendon dysfunction with flexor digitorum longus tendon transfer and calcaneal osteotomy. Orthopedics. 1996; 19(5): 383-388, indexed in Pubmed: 8727331.

19. O'Sullivan E, Carare-Nnadi R, Greenslade J, et al. Clinical significance of variations in the interconnections between 
flexor digitorum longus and flexor hallucis longus in the region of the knot of Henry. Clin Anat. 2005; 18(2): 121-125, doi: 10.1002/ca.20029, indexed in Pubmed: 15696523.

20. Pichler W, Tesch NP, Grechenig W, et al. Anatomical variations of the flexor hallucis longus muscle and the consequences for tendon transfer. A cadaver study. Surg Radiol Anat. 2005; 27(3): 227-231, doi: 10.1007/s00276005-0314-y, indexed in Pubmed: 15789138.

21. Razeghi M, Batt M. Foot type classification: a critical review of current methods. Gait Posture. 2002; 15(3): 282-291, doi: 10.1016/s0966-6362(01)00151-5, indexed in Pubmed: 11983503.

22. Romphothonga M, Traithepchanapai P. Sex determination through anthropometry of hand and foot in Thais. Chula Med J. 2019; 63(1): 47-55, doi: 10.14456/ clmj.1476.9.

23. Sangchay N. The soft cadaver (thiel's method): the new type of cadaver of department of anatomy, siriraj hospital. Siriraj Med J. 2014; 66(Suppl): S228-S231.

24. Shu Y, Mei Q, Fernandez J, et al. Foot morphological difference between habitually shod and unshod runners. PLoS One. 2015; 10(7): e0131385, doi: 10.1371/journal. pone.0131385, indexed in Pubmed: 26148059.

25. Sigvard T, Hansen J. Functional reconstruction of the foot and ankle. Lippincott Williams \& Wilkins, a Wolters Kluwer Business, Philadelphia 2000: 422-429.
26. Suttinark $P$, Suebpongsiri P. Clinical outcomes of flexor hallucis longus transfer for the treatment of Achilles tendinosis rupture. J Med Assoc Thai. 2009; 92 Suppl 6: S226-S231, indexed in Pubmed: 20120691.

27. Tashjian RZ, Hur J, Sullivan RJ, et al. Flexor hallucis longus transfer for repair of chronic achilles tendinopathy. Foot Ankle Int. 2003; 24(9): 673-676, doi: 10.1177/107110070302400903, indexed in Pubmed: 14524515.

28. Vasudha TK, Vani PC, Sankaranarayanan G, et al. Communications between the tendons of flexor hallucis longus and flexor digitorum longus: a cadaveric study. Surg Radiol Anat. 2019; 41(12): 1411-1419, doi: 10.1007/s00276-01902311-x, indexed in Pubmed: 31541272.

29. Wapner KL, Pavlock GS, Hecht PJ, et al. Repair of chronic Achilles tendon rupture with flexor hallucis longus tendon transfer. Foot Ankle. 1993; 14(8): 443-449, doi: 10.1177/107110079301400803, indexed in Pubmed: 8253436.

30. Williams D, McClay I. Measurements used to characterize the foot and the medial longitudinal arch: reliability and validity. Physl Ther. 2000; 80(9): 864-871, doi: 10.1093/ptj/80.9.864.

31. Wong CK, Weil R, de Boer E. Standardizing foot-type classification using arch index values. Physiother Can. 2012; 64(3): 280-283, doi: 10.3138/ptc.2011-40, indexed in Pubmed: 23729964.

32. Wulker N, Stephens MM, Cracchiolo A. An atlas of foot and ankle surgery. 2nd ed. Talor \& Francis, London 2005: 377-386. 